\title{
Alcohol-Pentazocine Combination: Implication on the Cytoarchitectural Profile of the Medial Prefrontal Cortex and Cerebellum of Juvenile Male Rats
}

\author{
*ADEKOMI DA, ADEWOLE OS, DARE BJ, OLANIYAN OO, OJEBISI RM, AJAO MS
}

Correspondence to Dr. Adekomi DA. Department of Anatomy, Osun State University, Osogbo, Osun State, Nigeria. Phone: +2348038441671. e-mail: adedayo.adekomi@uniosun.edu.ng

\begin{abstract}
Recently, Pentazocine and alcohol have become one of the drugs abused in the developing countries. However, there is the dearth of information on the effects of these substances on the cytological profile of the medial prefrontal cortex (MPFC) and cerebellar cortex; hence this study was aimed at evaluating the effect of these substances on the cytoarchitectural profile of the mPFC and cerebellar cortex of juvenile male rats. Twenty-four juvenile male rats were used for this study. They were randomly assigned to control (A), Alcohol-treated (B), Pentazocine-treated (C), and Pentazocine-Alcohol-treated group (D). Exposure to the various treatment paradigm was done s.c. twice daily (6hrs interval) for 14 days. It was observed that the cytological profile of the mPFC of the rats in the control groups was visible and well defined. In the $B, C$ and $D$ groups, there were numerous forms of neurodegeneration. There was also an increase in the density of astrocytes with the presence of glial scars. Furthermore, features of degenerative changes were also seen in the cerebellar cortex of the rats in the B, C, and D groups. It was observed from this study that exposure to Pentazocine-Alcohol combination triggers inflammatory and neurodegenerative processes in the cytoarchitectural profile of the mPFC and cerebellar cortex in juvenile male rats. These features could impair the functional integrity associated with these brain regions.
\end{abstract}

Keywords: addiction, substance abuse, opioid, male, young

\section{INTRODUCTION}

Non-medical use of legal or illicit (i.e. amphetamines, cannabis, caffeine, cocaine, hallucinogens, nicotine, opiates, psychoactive inhalants, and many other hypnotics, sedatives, and stimulants) drugs/substances have been abused and/or misused for several reasons which could either be for pleasure or the avoidance of pain. Continuous misuse of drugs often results into addiction which appears as an extreme compulsory-desire for the drug with a diminished ability to limit the desire to take the $\operatorname{drug}(\mathrm{s})$, even at the expense of life-threatening adverse repercussions (WHO, 2004). Pentazocine (PTZ) is a non-narcotic analgesic, structurally related to morphine, which is widely used in the management of patients with acute or chronic pain. However, over the years, PTZ has been suggested to be a substance of abuse which might have a deleterious effect on the brain (Gould, 1972; Waldmann and Horsfall, 1977; King and Betts, 1978). The developing countries are still battling the menace of drug abuse. For instance, in a study carried out in Nigeria by the United Nations Office on Drugs and Crime (UNODC, 2012), it was observed that out of the 1,147 street injectors recruited into the survey, $8 \%$ were current injectors and $13 \%$ had injected at least once in the past (Adelekan and Lawal, 2006; Onifade et al., 2011). The drugs most often injected were

Submitted $7^{\text {th }}$ June 2017, revised on $7^{\text {th }}$ September 2017. Published online $12^{\text {th }}$ Nov 2017. To cite: ADEKOMI DA, ADEWOLE OS, DARE BJ, OLANIYAN OO, OJEBISI RM, AJAO MS. Alcohol-Pentazocine Combination: Implication on the Cytoarchitectural Profile of the Medial Prefrontal Cortex and Cerebellum of Juvenile Male Rats. Anatomy Journal of Africa. 2017. Vol 6 (3): $1080-1091$. 
heroin, cocaine, pentazocine and speedball (a combination of heroin and cocaine)' (Obot, 2013). Few studies reported the abuse of PTZ, maybe because of the diminished usage of the drug in many of the developed countries of the world. However, PTZ addiction persists in underdeveloped countries of West Africa, where PTZ continues to be prescribed by physicians in the clinical management of pain (Mudrick et al., 2011; Iheanacho et al., 2015; Armiya'u et al., 2016).

Adverse interactions have been observed between alcohol and some class of ethically prescribed drugs such as anesthetics, antibiotics, anticoagulants, anticonvulsants, antidepressants, antidiabetics, antihistamines, antipsychotics, antiulcer agents, cardiovascular agents, narcotic and non-narcotic pain relievers, as well as sedatives (Adams, 1995; Hersh et al., 2007). Pentazocine is a narcotic agonist/antagonist analgesic derived from benzmorphinan. Lang and Pilon (1980) reported that PTZ produces minimal euphoria but has some dysphoric effect in the insignificant number of individuals. It was initially thought to be non-addictive ethical medication, but its potential for abuse and dependence was soon observed (Weber and Fromme, 1970; Huskisson, 1974; Makanjuola and Olatunji, 2009). Since then, various 'pharmaco-medicolegal' efforts have been made to significantly minimize its abuse and dependence (Makanjuola and Olatunji, 2009). Incidences of drug abuse in Nigeria has persisted from the 1960s till date (Asuni, 1964; Lambo, 1965; Adelekan et al, 2000; Adamson et al., 2010; Onyecho et al., 2016). Following these reports, other drugs of abuse such as alcohol, tobacco, cannabis, stimulants, cocaine, heroin, and hallucinogens have also been documented (Makanjuola and Olatunji, 2009). However, in basic research, there is the dearth of data on the abuse of PTZ and alcohol. Despite the available evidence on the abuse of PTZ, its potentials effects with alcohol on body weight gained, astrocytic reactions, neuronal energy metabolism, apoptosis and neuronal morphology are yet to be elucidated. The aim of the present study was to examine and analyze the neuropathological features in the medial prefrontal cortices of juvenile male rats exposed to alcohol and/or PTZ.

\section{MATERIALS AND METHODS}

\section{Animal Care and Ethical Approval}

The experiments were carried out in adherence to the guidelines of the Health Research Ethics Committee (College of Health Sciences, Osun State University, Osogbo, Nigeria). Juvenile male Wistar rats (post-natal day 28) were obtained from the animal facility of the College of Health Sciences, Osun State University (Osogbo, Nigeria) and were transferred to the experimental rooms. The rats were housed in stainless steel cages with a natural light/dark cycle paradigm. The temperature of the room was maintained at $24 \pm 1^{\circ} \mathrm{C}$ with a humidity of $53 \pm 12 \%$, for at least 7 days before they were exposed to the experimental paradigm. The rats were fed standard rat pellets and drinking water was provided ad libitum. In this experiment, sufficient efforts were made to minimize the numbers of rats used, as well as pains, and suffering. During this study, there was no animal mortality.

\section{Chemicals and Reagents}

The PTZ and alcohol used in this study were kindly provided as gifts from Dr. Adewole (Department of Anatomy and Cell Biology, Obafemi Awolowo University, Nigeria) and Mr. Olaniyan (Department of Medical Biochemistry, Osun State University, Nigeria) respectively.

\section{Experimental Animals and Treatment}

The experiment was performed on juvenile male Wistar rats $(N=24)$. The rats were randomly assigned into 4 groups designated as $\mathrm{A}, \mathrm{B}, \mathrm{C}$, and $\mathrm{D}$. The rats in group $\mathrm{A}(n=6$; control) were treated with double distilled water; rats in group $\mathrm{B}(n=6)$ were exposed to 
$1.0 \mathrm{ml}$ of $15 \%$ ethanol; the rats in group C ( $n=$

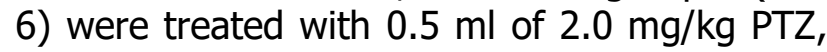
while the rats in group $\mathrm{D}(n=6)$ were cotreated with $1.0 \mathrm{ml}$ of $15 \%$ ethanol and $0.5 \mathrm{ml}$ of $2.0 \mathrm{mg} / \mathrm{kg}$ PTZ. All administrations were done twice daily at an interval of 6 hours apart. Twenty-four hours after the administration of the last doses, the rats were decapitated. Using the Paxinos and Watson atlas (Paxinos and Watson, 2006) and Leica dissecting microscope, the mPFC (4.70-2.70 mm ventral and 4.70$2.70 \mathrm{~mm}$ dorsal to the bregma) was identified and isolated. The cerebellar cortices were excised from the whole brain. These were subsequently paraffin-embedded and sectioned at $5 \mu \mathrm{m}$ on a microtome respectively for routine histochemical and immunohistochemical procedures using Cresyl violet and glial fibrillary acidic protein stains respectively.

\section{Histochemical Procedure}

For the histological and histochemical procedures, the mPFC and cerebellar cortex were fixed in $10 \%$ neutral buffered formalin. 24 hours after fixation, specimens were dehydrated in an ascending grade of alcohol, cleared in four changes of isopropanol (3 $\mathrm{min}$ $45 \mathrm{sec}$ ) using an automatic tissue processor (Leica ASP 200S, Germany) and embedded in molten paraffin. Sections of 5 microns thickness were cut using rotary microtome (Leica RM 2135, Germany) and mounted on clean slides. The sections were stained with Cresyl fast violet stains (Bancroft and Gamble, 2002).

\section{Immunocytochemistry of GFAP}

Immunocytochemical investigation of Glial fibrillary acidic protein (GFAP) immunocytochemistry was performed on the sagittal paraffin-embedded section of the MPFC and cerebellum. The dissected mPFC and vermis from the cerebella were post-fixed overnight at $4^{\circ} \mathrm{C}$ in $4 \%$ paraformaldehyde, deparaffinized, rehydrated and incubated for 30 min at room temperature with $3.0 \%$ hydrogen peroxide. The processed $\mathrm{mPFC}$ and cerebellar were rinsed in phosphate buffered saline (PBS) and incubated overnight at $4^{\circ} \mathrm{C}$ with anti-GFAP antibodies (Elabscience, USA). The tissue sections were then rinsed with PBS, incubated with biotinylated peroxidase-conjugated secondary antibody for $1 \mathrm{~h}$. For visualization of the immunogenic reactions, the tissue sections were subsequently rinsed in PBS and incubated with diaminobenzidine tetrachloride (DAB). The sections were then rinsed in PBS, counterstained with hematoxylin, dehydrated and mounted. The negative control sections were incubated with normal serum in lieu of the primary antibody, while other protocol remains the same (Machado and Alessi, 1997; RamosVara et al., 2008; Fang et al., 2014; Mousa and Shehab, 2015).

Photomicrography: Images of the sections of the mPFC and cerebellum processed for histochemical, and immunohistochemical observations were captured using Zeiss Axio Scope.A1 with a cameroscope (AxioCam MRc) connected to a computer interface.

\section{Cresyl Violet Stain for Nissl substances}

The sections of the MPFC and cerebellar cortex of control rats (A) stained with Cresyl violet displayed the well-known cortical organization. In the mPFC, the neurons are with normal appearance, and uniformly dispersed smallsized neuroglia cells interspaced within the neurophil. The granular, Purkinje cell, and molecular layers as well as prominent extra granular layer (EGL) composed of the

\section{RESULTS}

proliferative and premigatory zones were seen in the cytoarchitectural profile of the cerebellar cortex. In the alcohol treated group (B), numerous pyknotic cells and apoptotic bodies were predominantly found in the $\mathrm{MPC}$, and in the outer proliferative zone of the EGL, and only occasional isolated cell death figure were detected in the internal granular layer (IGL) of the cerebellar cortex. There were prominent dying cells in the Purkinje cell-Bergmann glia 
layer. The cortical cerebellar architecture in this group of rats were not preserved. Similar neurodegenerative features were observed in the cytoarchitecture of the MPFC and cerebellar cortex of the rats in the Pentazocine treated group (C). In the mPFC, many of the neuronal cells are with prominent cytoplasmic vacuolation, fragmented cytoplasm, with activeappearing microglial cells. The light microscopy of the cortical layers of the cerebellum of the rats in this group revealed numerous dying cells with the typical morphological features of apoptosis. The section of the MPFC of the Pentazocine and alcohol co-treated group (D) showed neurons with distorted outline, swollen and vacuolized cytoplasm. The neurons are with karyorrhectic nuclei. Extensive cell death was seen in the cortical layers of the cerebellum of the rats in this group (Figs. 1-2).
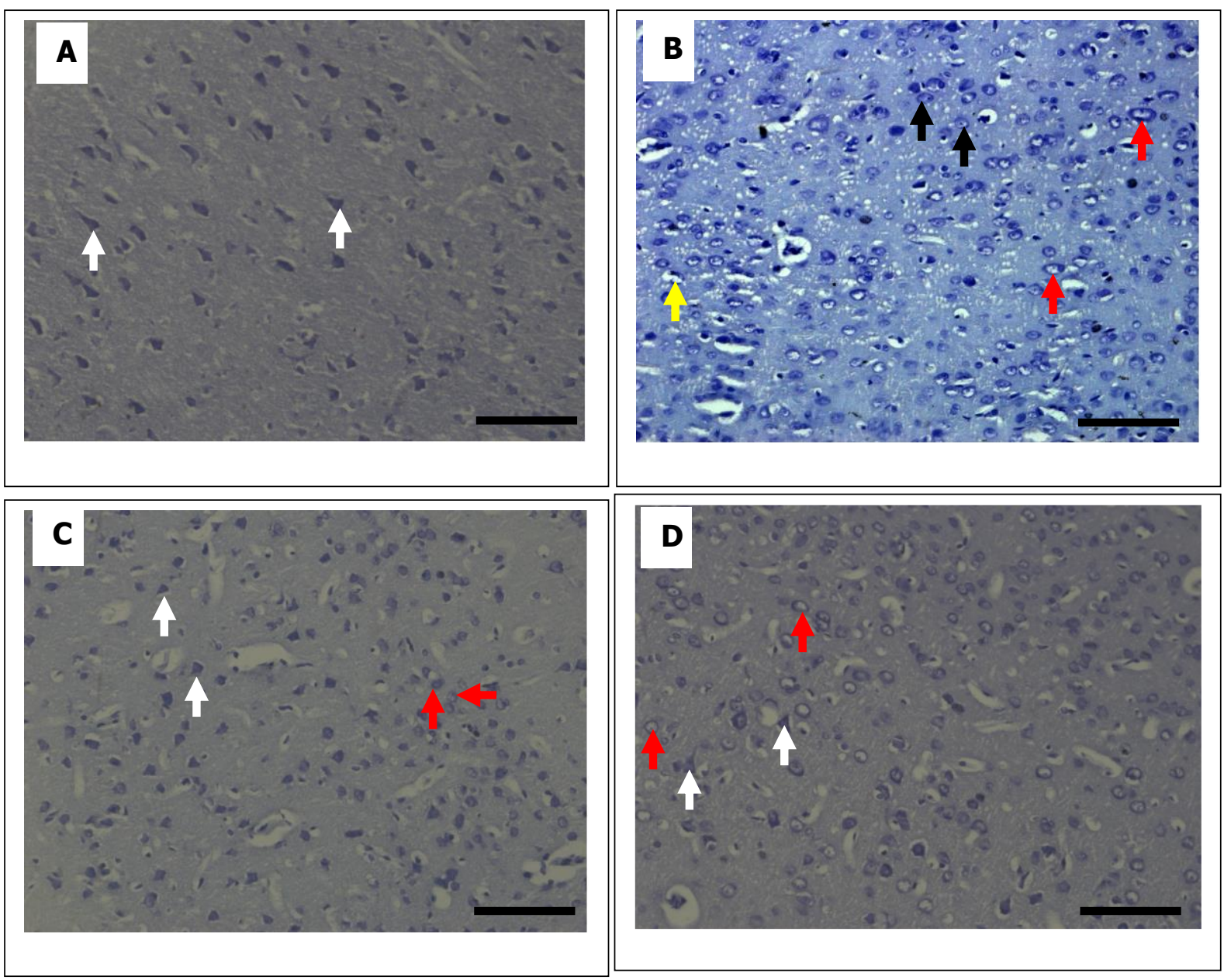

Fig. 1: Cresyl violet stained photomicrographs of the mPFC of the rats in the control (A), alcohol (B), pentazocine $(\mathbf{C})$, and pentazocine + alcohol (D) treated groups. The neurons in the control group appear normal with no perineuronal cavitation; the Nissl substances were within the neurons, and are with intact cytoplasmic contents (white arrows); neurons with features of chromatolysis, fragmented cytoplasm and perinuclear Nissl deposits (red arrows), pyknotic neurons (black arrows), and neuron with ruptured membrane

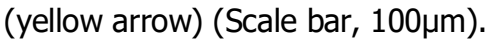



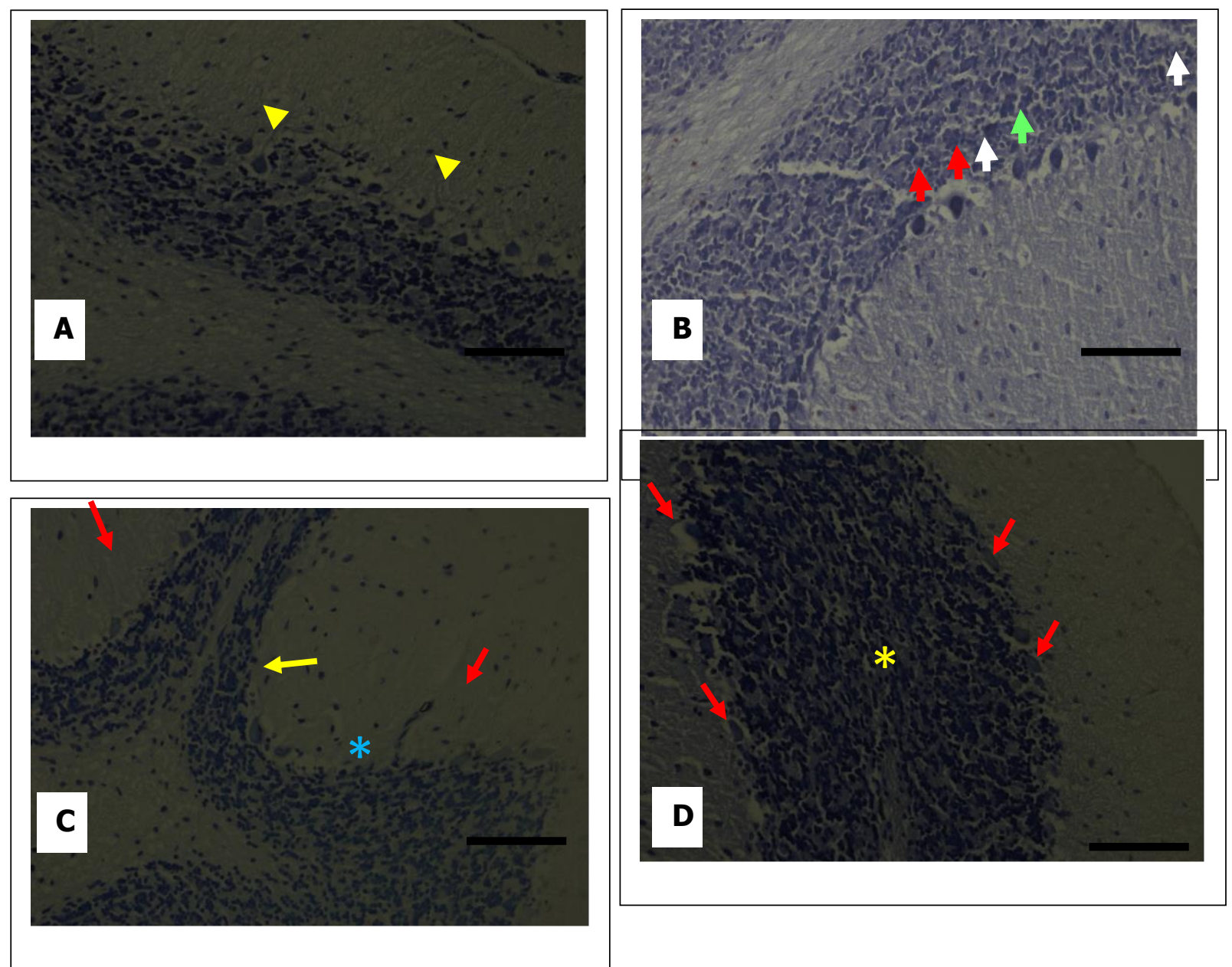

Fig. 2: Cresyl violet demonstration of Nissl's substances in the cerebellar cortex of the representative rat in the control $(\mathbf{A})$, alcohol $(\mathbf{B})$, pentazocine $(\mathbf{C})$, and pentazocine + alcohol (D) treated groups. In the control, the distribution of Nissl's substances in the three prominent layers of the cerebellum was heterogenous. There was a dense net of Cresyl violet stained Purkinje neurons with intensively stained cell bodies and elongated processes extending into the molecular layer (yellow arrowheads); in B, there was a slightly weak expression of Cresyl violet stain in the molecular layer, though the Purkinje neurons are deeply stained, they have lost their processes (red arrows). Note the pyknotic Purkinje neuron (green arrow), and vacuolated Purkinje neuron (white arrow); in C and D, the Purkinje neurons are sparsely stained with Cresyl violet stain; the Purkinje neurons have lost their neuronal processes and are vacuolated with degraded cytoplasmic contents (red arrows). Note the Purkinje neuron with an extended process (yellow arrowhead) and the pyknotic Purkinje neuron (green arrow). The distribution of Nissl substances in the granular layer are well preserved (blue and yellow asterisks) (Scale bar, 100 $\mu \mathrm{m})$.

\section{Anti-GFAP Immunocytochemistry in the MPFC}

The anti-GFAP immunocytochemical procedure provides a marked positive marker for the demonstration of astrocytes and their processes in the CNS. The expression of GFAP in the astrocytes in the mPFC of the rats in the $B, C$, and $D$ groups showed that the administration of these substances confers degenerative changes on the cytoarchitectural profile of the rats in the respective groups compared with the control (A) (Fig. 3). Five (5) of the 6 rats in group B displayed abundant GFAP immuno-positive astrocytes with intensively stained cell bodies 
(yellow arrows) and elongated astrogliotic processes with scar formation (red circles), three (3) of the 6 rats in group C has elevated astrocytic density with prominent astroglial scar (red circle) and marked population of neuronal cell death, and in group $D$, there was a sparse population of GFAP immuno-positive astrocytes, however, patches of glia scar (red circle) were present in the sections of the mPFC of all the rats in this group. The mPFC of the rats in this group has the highest physical population of neuronal cell death. These are more prominent than any neuropathological deviations seen in this group.
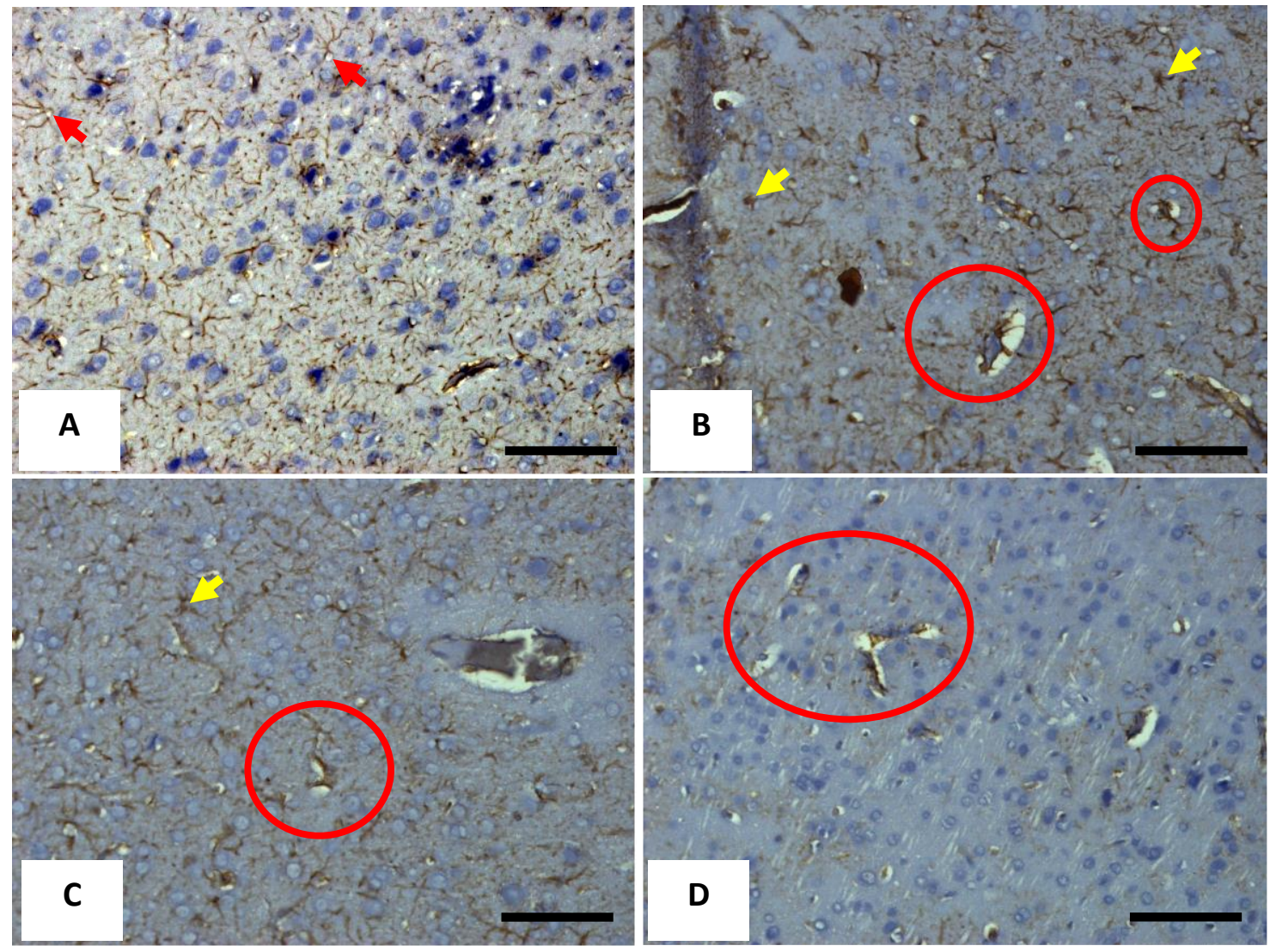

Fig. 3: Anti-GFAP antibody labelled sections of the mPFC of the rats in the control (A), alcohol (B), pentazocine (C), and pentazocine + alcohol (D) treated groups. There was a uniform distribution of GFAP immuno-positive reactivity in the mPFC of the rat in the control group showing astrocyte with normal cytological features (red arrows). In the alcohol treated group, there were more GFAP immuno-positive astrocytes with intensively stained cell bodies (yellow arrowheads) and elongated astrogliotic processes with scar formation (red circles). In group C, increased astrocytic density was observed with prominent astroglial scar (red circle) and marked neuronal loss. In group D, there were patches of glia scar appearance, and severe diffuse reactive astrogliosis; these are more prominent than any neuropathological deviations present in this group (Scale bar, $100 \mu \mathrm{m})$. 

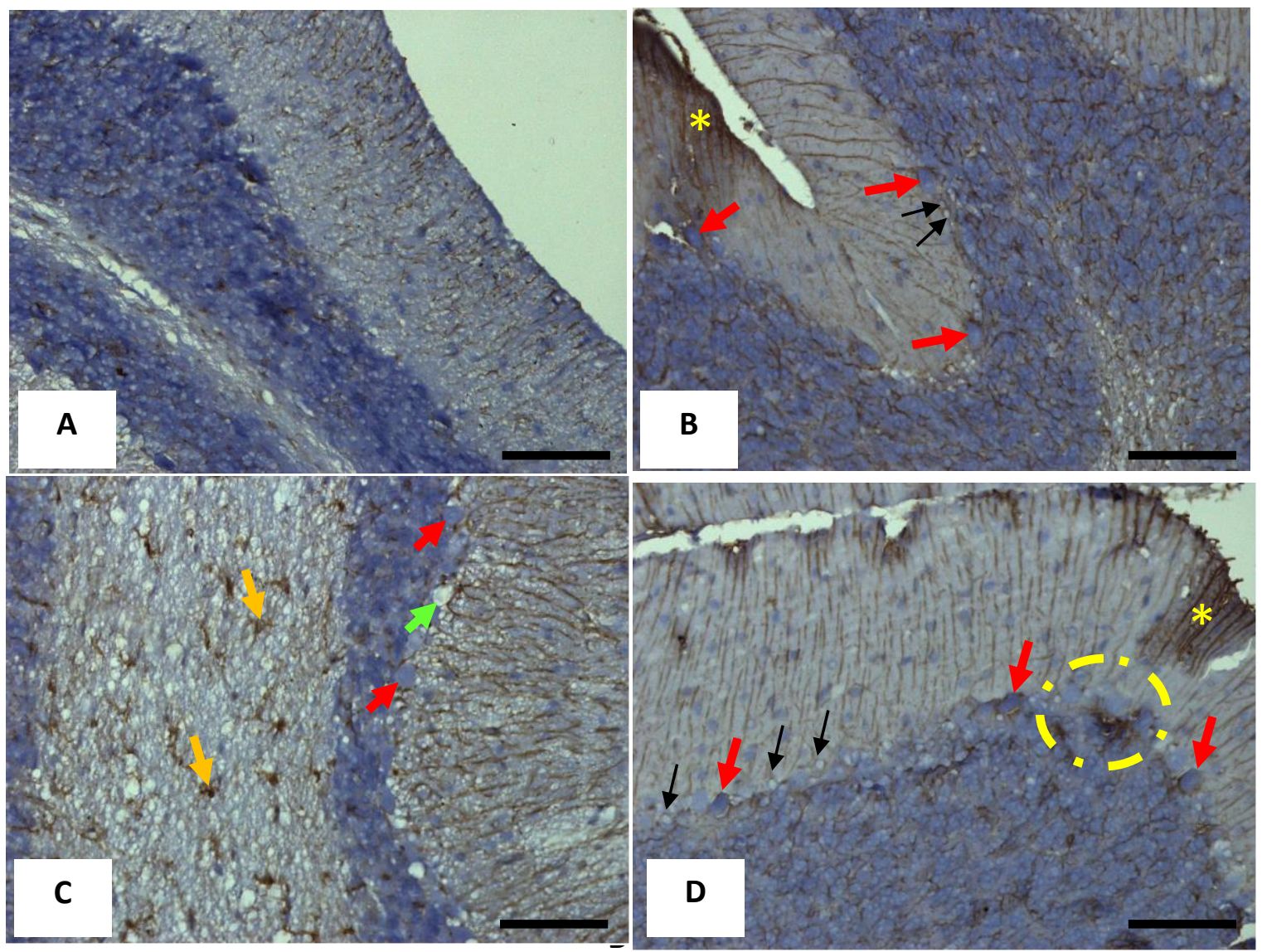

Fig. 4: GFAP immunocytochemistry of the cerebellar cortex in the control (A), alcohol (B), pentazocine (C), and pentazocine + alcohol (D) treated groups. In A, prominent granular cells are visible. The cortical cytological layers of the cerebellar cortex in this group are well defined. In B, slight increased intensity of GFAP signaling was seen compared with the control, numerous features of cell death were also observed in the Purkinje cell layer (red arrow heads) and external aspect of the internal granular layer. Note the smooth immunostaining of the processes (Bergmann glia) extending out through the molecular layer in both groups (prominent dark-brown patches of these bands were seen in the alcohol treated rat [yellow asterisk]; the presence of pyknotic Purkinje cells (presumably undergoing apoptosis) surrounded by GFAP immuno-positive Bergmann glial processes (red arrow) and internal granular layer (black arrow). In C, the Purkinje cells are undergoing degenerative processes (vacuolation; green arrow, pyknosis; red arrows); extensive arborization of astrocytic processes and the intense reactivity for GFAP along the glia limitans were observed in the molecular layer (orange arrows) compared with the control. In $\mathbf{D}$, there is the presence of glial scar (yellow dashed circle). Also seen in this group is the immunostaining of the Bergmann glia radiating through the molecular layer in this group [yellow asterisks]; the presence of pyknotic Purkinje cells (presumably undergoing apoptosis) surrounded by GFAP immunopositive Bergmann glial processes (red arrow) and vacuolation in the internal granular layer (black arrows) (Scale bar, $100 \mu \mathrm{m})$.

\section{Anti-GFAP Immunocytochemistry in the cerebellar cortex}

In the control rats, the cytological layers with prominent cells in the cerebellar cortex were visible and well defined in the granular, Purkinje cell, and molecular layers. In group B, a slight intensity of GFAP signaling was seen compared with the control. Many vacuolated and pyknotic cells and apoptotic bodies were seen both in the Purkinje cell (red arrowheads) and external 
zone of the internal granular layer. In group C, there was a complete loss of cells in the molecular layer. There was the presence of smooth immunostaining of the Bergmann glia processes extending out through the molecular layer in both groups. These were present as prominent bands of a dark-brown patch (yellow dotted circle) in group $D$; the presence of pyknotic Purkinje cells presumably undergoing apoptosis surrounded by GFAP immuno-positive Bergmann glial processes (red arrow) and internal granular layer (black arrow) were also observed (Fig. 4).

\section{DISCUSSION}

In the developing nations, several medico-legal over-the-counter drugs are abused. Many of these substances are abused concomitantly with many of other substances.

Several studies have documented the dysfunctions in the morphology and functional integrities of the cells in the subregions of the PFC following exposure to abused substances such as alcohol (Lasseter et al., 2010; Bossert et al., 2013; Bull et al., 2015). This is the first study showing neuronal integrity and GFAP expression in the MPFC and cerebellum of juvenile male rats following exposure to Pentazocine and/or Alcohol. Nissl staining is a rapid and simple staining technique for examining the morphology of neuron undergoing degeneration suggestive of apoptosis. In agreement with the integrity of Cresyl violet as a marker of neuronal degeneration in the CNS, we observed that the neurons in the MPFC and cerebellar cortex of the juvenile male rats treated with alcohol, Pentazocine, and Pentazocine + alcohol are undergoing neurodegeneration with peripheral deposit of Nissl substances and chromatolysis suggesting that the neurons are undergoing apoptotic form of cell death (Figs. 1-2).

We further observed that exposure to concomitant or either of these substances mediated specific alterations in the of GFAPexpressing astrocytes within the MPFC and the cerebellar cortex of the treated rats (Fig. 3).

The GFAP immunocytochemical staining provided confirmation of the degeneration of the Purkinje cells in the alcohol-treated group.
This antibody, present in the astrocytes showed Bergmann glial processes around the degenerating Purkinje cells bodies. The Purkinje cells in the respective groups are surrounded by the debris of Bergmann process suggesting apoptotic processes (Fig. 4) compared with the Purkinje cells in the control group devoid of debris of Bergmann debris and feature of apoptosis. This is similar to the outcome of the study of Xu et al (2000).

Bands of strong positive GFAP immunostaining represents increased activities of astrocytosis by Bergmann glia, increased the population of neuronal perikarya and neuronal cell death, degenerating Purkinje cell and granular cells detectable in the alcohol, Pentazocine, and Pentazocine + alcohol-treated groups. Some of the bands of the Bergmann processes are occasional densely stained with the GFAP immuno-marker (Fig. 4). Our study showed that abuse of Pentazocine could be deleterious to the functional integrity of the cerebellar cortex in juvenile subjects. This is in support of the report of Luo (2015),

Our study did not indicate any observable alteration in the morphology of the Bergmann processes but induces a response pattern of reactive gliosis and a common pattern of association with dying cells in the Purkinje and outer aspect of the internal granular layers. Reactive Bergmann glial cells is seen in this study surrounding dying Purkinje cells. This further confirms that these Bergmann cells are involved in the separation of apoptotic cells before their removal by phagocytosis. This cleansing mechanism by the Bergmann glial 
cells may be a form of protection for the surrounding healthy and viable cells from the latent harmful effects of the dying cells.

Alcohol administration elevate the expression of GFAP in the mPFC and cerebellum of the treated rats. This observation was in consonant with the published outcome of the study of Dalcik et al. (2009) and Udomuksorn et al (2011) who observed that astrocytes are a major target of alcohol in the human brain. Miguel-Hidalgo (2005), observed that the density of GFAP-expressing astrocytes is lower in the prelimbic prefrontal cortex of alcoholnaïve rats bred for high alcohol preference over water. Bull et al (2014) further reiterated that in the nucleus accumbens core, changes in astrocyte density were only seen after 3-week abstinence, and this increase positively correlated with the motivation to resume ethanol self-administration after abstinence. Wilhelm and Guizzetti (2015), suggested a reduction in cell number has been reported for several glial cell types in animal models of fetal syndrome.

Astrocytes performs significant roles in the functional regulation of defense against oxidative and toxic insults around the neurons and synapses in the CNS microenvironment (Sofroniew and Vinters, 2010). Astrocyte activation has been reported to be initiated by environmental toxicant (Block and Hong, 2007) and may experience a morphological modification with short or non-existent processes to permit phagocytosis. Hence, we presume that in the present study, upregulation of glial cells in the expression of GFAP in the mPFC and cerebellar sections could be suggestive of neurodegenerative effects induced by alcohol.

The alterations observed in the GFAP expressing cells following concomitant exposure of the juvenile rats to Pentazocine and alcohol had a prominent effect on the formation of glial scars (Fig. 4) suggesting the exposure to these substances can degrade the axon thereby leading to the glial scar. According to Fawcett and Asher (1999), impaired axonal regeneration or damage to the axons in the CNS will often lead to the formation of glial scar. Five types of cells namely astrocytes, meningeal cells, microglia, oligodendrocyte precursors, and oligodendrocytes make up this scar. Many of these cells have inhibitory functions (except the microglia) and are capable of inhibiting many of the CNS axons, although astrocytes are plastic in nature and can perform growth-performing or growth inhibiting functions under different responses to stimuli. The scar formation seen in this study could be the growth inhibiting functions of the astrocytes during exposure to these respective substances.

The deleterious effects of alcohol, Pentazocine, and Pentazocine + alcohol indicates that these substances induce their various neurodegenerative effects through oxidative stress with the presence of reactive oxygen species which could have elicited the activated astroglia reaction, and neuronal cell death (Krasnova and Cadet, 2009)

Pow (2001) and Wigley et al., (2007) reported that glial cells positive to the immunoexpression of GFAP in the mPFC contain cystine/glutamate antiporters that maintain extracellular levels of non-synaptic glutamate and provide functional support to neurons by regulating extracellular potassium and the reuptake of glutamate at synapses. Astrocytes play several biological roles in the regulation of synaptic transmission. Astrocytic control of glutamatergic signaling during abstinent periods may also critically impact reinstatement of drug-seeking behaviors (Turner et al., 2013).

In this study, we observed marked astrocytic reactions in the MPFC and cerebellar cortex of the treated rats vis-à-vis the control (Figs. 3-4). The mPFC is a key component of the mesocorticolimbic system that is assumed to influence drug-taking behavior (Kalivas, 2008). Exposure to alcohol or opioids have been reported to cause partial inactivation and lesion of the prefrontal cortex and these alterations have been linked to neuronal loss and impairment behavioral sensitization (Pastor et al., 2005). 
There was a decrease in the density of the neurons in the MPFC of the rats exposed to; alcohol, Pentazocine, and Pentazocine-Alcohol treated groups. This is suggestive of the neurotoxic effects of these substances. In this study, we cannot ascertain if the changes in the population of the astrocytes occurred prior the neuropathological deviations or were initiated as a form of adaptive response to the loss of the neurons.

Cox-Limpens et al., (2015) and Kubesova et al. (2015) suggested that the mechanism by which astrocytes become reactive astrogliosis is characterized by rapid synthesis of GFAP in the presence of oxidative stress and/or in cases of inflammation. Several molecular studies have shown that astrogliosis in many neurodegenerative disorders is one of the crucial process associated with oxidative stress (Bush et al. 1999; Santos and Saraiva 2004; Ribotta et al. 2004), therefore, it is inferred that the observed astrogliosis and glial scar formation was seen in the MPFC of the rats in the $B, C$ and $D$ groups are a result of the respective treatment paradigms. These observations are suggestive of the deleterious hyper interactions between opioid receptors and alcohol.

In the cerebellum, the marked presence of reactive gliosis of Bergmann glial cells, diffused astrocytosis, ablation, and death of granular cells. These features are suggestive of the deterioration of cellular functions which ultimately leads to the destruction of cellular structures and cell death. Following the coadministration of Pentazocine and alcohol, the Bergmann glial cell process is oedematous and severally distorted. This observation is similar to the reported outcome of Bekheet et al., (2010) that opioid administration impairs the morphology of Purkinje cells, triggers significant degeneration of granular cells, and destruction of inner mitochondria membrane.

Ethanol is shown to disrupt the molecular events at the mossy fiber-granule cell - Golgi cell (MGG) synaptic site and granule cell parallel fibers - PCs (GPP) synaptic site, which may be responsible for ethanol-induced cerebellar ataxia (Luo, 2015). Observations from this study were also in consonant with the findings of Cservenka et al., (2015) who reported deuced cerebellar brain activities in adolescent binge drinker. The aggravated impact seen in the Pentazocine + alcohol treated group could possibly be as a result of the effects of the interaction of Pentazocine + alcohol exposure on the neurotransmitters responsible for cerebellar functions.

In conclusion, we have shown that exposure to Pentazocine-Alcohol can cause cytological degeneration in cytoarchitectural profile of the mPFC (increased astrocytic density and scar formation) and cerebellar cortex (degeneration of Purkinje cells and granular cells) of juvenile male rats. We therefore recommend that a time course response of the astrocytes and Bergmann cells in the cerebellar cortex and the scar formation in the MPFC be further evaluated using multiple immunomarkers to study their relativity to Pentazocine-Alcohol neurotoxicity at different time interval as this may provide new pharmacological method that will enhance neuronal recovery and enhance restorative processes by modulating astrogliosis in instances of restorative procedure for individuals addicted to these substances.

\section{REFERENCES}

1. Adams WL. 1995. Interactions between alcohol and other drugs. Int J Addict 30:1903-23

2. Adamson TA, Onifade PO, Ogunwale A. 2010. Trends in sociodemographic and drug abuse variables in patients with alcohol and drug use disorders in a treatment facility. West Afr J Med 29(1):12-18

3. Adelekan M, Lawal R. 2006. Drug use and HIV infection in Nigeria: a review of recent. Afr J Drug Alchol Studies 5(2):118-129 
4. Adelekan ML, Ndom RJE, Makanjuola AB, Parakoyi DB, Osagbemi GK, Fagbemi O et al. 2000. Trends analyses of substance use among undergraduates of University of Ilorin, Nigeria, 1988-1998. Afr J Drug Alcohol Studies 1:39-52

5. Armiya'u AY, Garba BI, Haliru DG. 2016. Intravenous pentazocine dependence in a young sickle cell anemia patient: A case report. Case Rep Int. 5:1-4

6. Asuni T. 1964. Socio-psychiatric problems of drug abuse in Western Nigeria. Afr J Psych 16:17-28

7. Bekheet SH, Saker SA, Younis AEA. 2010. Histopathological and biochemical changes of morphine sulphate administration on the cerebellum of albino rats. Tiss Cell 42(3):165-175

8. Block ML, Hong JS. 2007. Chronic microglial activation and progressive dopaminergic neurotoxicity. Biochem Soc Trans 35:1127-1132

9. Bossert JM, Marchant NJ, Calu DJ, Shaham Y. 2013. The reinstatement model of drug relapse: recent neurobiological findings, emerging research topics, and translational research. Psychopharmacol. 229:453-76

10. Bull C, Freitas KC, Zou S, Poland RS, Syed WA, Urban DJ, et al. 2014. Rat nucleus accumbens core astrocytes modulate reward and the motivation to self-administer ethanol after abstinence. Neuropsychopharmacol 39:2835-2845

11. Bull C, Syed WA, Minter SC, Bowers MS. 2015. Differential response of GFAP-positive astrocytes in the rat prefrontal cortex following ethanol self-administration. Alcohol Clin Exp Res 39(4):650-658

12. Bush T, Puvanachandra N, Horner C, Polito A, Ostenfeld T, Svendsen C et al. 1999. Leukocyte infiltration, neuronal degeneration, and neurite outgrowth after ablation of scar forming, reactive astrocytes in adult transgenic mice. Neuron 23(2):297-308

13. Cox-Limpens K, Strackx E, Van den Hove D, Van Ekkendonk JR, de Jong M, Zimmermann LJ et al. 2015. Fetal asphyctic preconditioning protects against perinatal asphyxia-induced apoptosis and astrogliosis in neonatal brain. CNS Neurol Disord Drug Targets 14(1):33-40

14. Cservanka A, Jones SA, Nagel BJ. 2015. Reduced cerebellar brain activity during reward processing in adolescent binge drinkers. Dev Cog Neurosci 16:110-120

15. Dalcik H, Yardimoglu M, Filiz S, Gonca S, Dalcik C, Erden BF. 2009. Chronic ethanol-induced glial fibrillary acidic protein (GFAP) immunoreactivity: an immunocytochemical observation in various regions of adult rat brain. Int J Neurosci. 119:1303-1318

16. Fan S, Hao Z, Zhang L, Chen X, Zhou J, Zang Y, Tai S, Liang C. 2014. Increased chromogranin A and neuron-specific enolase in rats with chronic nonbacterial prostatitis induced by 17-beta estradiol combined with castration. Int J Clin Exp Pathol 7(7):3992-3999

17. Fawcett JW, Asher RA. 1999. The glial scar and central nervous system repair. Brain Res Bull 49(6):377-391

18. Gould WM. 1972. Central nervous disturbance with pentazocine. Br Med J. 1(5795):313-314

19. Hersh EV, Pinto A, Moore PA. 2007. Adverse drug interactions involving common prescription and over-the-counter analgesic agents. Clin Ther 29:2477-2497

20. Iheanacho OE, Ezenwenyi IP, Enosolease ME. 2015. Pentazocine abuse in Sickle Cell Disease Patients Seen at a Tertiary Hospital in Nigeria: A Chronic Menace. Int J Tropical Dis Health. 9(1):1-8

21. Kalivas P. 2008. Addiction as a pathology in prefrontal cortical regulation of corticostriatal habit circuitry. Neurotox Res 14:185-189

22. King A, Betts TA.1978. Abuse of pentazocine. Br Med J 2(6129):21

23. Krasnova IN, Cadet JL. 2009. Methamphetamine toxicity and messengers of death. Brain Res Rev 60:379-407

24. Kubesova A, Tejkalova H, Syslova K, Kacer P, Vondrousova J, Tyls F et al. 2015. Biochemical, histopathological and morphological profiling of a rat model of early immune stimulation: relation to psychopathology. PLoS One 10(1):e0115439

25. Lambo TA. 1965. Medical and social problems of drug addiction in West Africa, with special emphasis on psychiatric aspects. Bull Narc 17:3-13 
26. Lasseter HC, Xie X, Ramirez DR, Fuchs RA. 2010. Prefrontal cortical regulation of drug seeking in animal models of drug relapse. Curr Top Behav Neurosci 3:101-17

27. Luo J. 2015. Effects of ethanol on the cerebellum: advances and prospects. Cereb 14(4):383-385

28. Luo J. 2015. Effects of ethanol on the cerebellum: advances and prospects. Cereb 14(4):383-385

29. Makanjuola AB, Olatunji PO. 2009. Pentazocine abuse in Sickle Cell Anaemia Patients: A Report of Two Case Vignettes. Afr J Drug and Alcohol Studies 8(2):59-64

30. Miguel-Hidalgo JJ. 2005. Lower packing density of glial fibrillary acidic protein-immunoreactive astrocytes in the prelimbic cortex of alcohol-naive and alcohol-drinking alcohol-preferring rats as compared with alcohol-nonpreferring and Wistar rats. Alcohol Clin Exp Res 29:766-772

31. Mudrick C, Isaacs J, Frankenhoff J. 2011. Case Report: Injectable pentazocine abuse leading to necrotizing soft tissue infection and florid osteomyelitis. Hand 6(4):457-459

32. Obot IS. 2013. Prevention and Treatment of Drug Dependence in West Africa. WACD Background Paper No. 21.

33. Onyencho VC, Pindar SK, Mshelia AA, Ibrahim AW, Wakil AM, Balogun AG. 2016. A Report of Personality Disposition and Pentazocine Abuse in Sickle Cell Anemia Patients: A report of two cases. Pyrex J Med Med Sci 3(1):001-004

34. Pastor R, Aragon CM. 2005. The Role of Opioid Receptor Subtypes in the Development of Behavioral Sensitization to Ethanol. Neuropsychopharmacol 31:1489-1499

35. Pow DV. 2001. Visualising the activity of the cystine-glutamate antiporter in glial cells using antibodies to aminoadipic acid, a selectively transported substrate. Glia 34:27-38

36. RibottaMG, Menet V, Privat A. 2004. Glial scar and axonal regeneration in the CNS: lessons from GFAP and vimentin transgenic mice. Acta Neurochir 89:87-92

37. Santos SD, Saraiva MJ. 2004. Enlarged ventricles, astrogliosis and neurodegeneration in heat shock factor 1 null mouse brain. Neurosci 126(3):657-63

38. Sofroniew MV, Vinters HV. 2010. Astrocytes: biology and pathology. Acta Neuropathol 119:7-35

39. Turner JR, Ecke LE, Briand LA, Haydon PG, Blendy JA. 2013. Cocaine-related behaviors in mice with deficient gliotransmission. Psychopharmacol 226:167-176

40. Udomuksorn W, Mukem S, Kumarnsit E, Vongvatcharanon S, Vongvatcharanon U. 2011. Effects of alcohol administration during adulthood on parvalbumin and glial fibrillary acidic protein immunoreactivity in the rat cerebral cortex. Acta Histochem 113:283-289

41. United Nations Office on Drugs and Crime (2012). World Drug Report 2012. Vienna: UNODC.

42. Waldmann E, Horsfall PA. 1977. Pentazocine addiction: a warning. Br Med J. 1(6061):642.

43. Wigley R, Hamilton N, Nishiyama A, Kirchhoff F, Butt AM. 2007. Morphological and physiological interactions of NG2-glia with astrocytes and neurons. J Anat 210:661-670

44. Wilhelm CJ, Guizzetti M. 2015. Fetal Alcohol Spectrum Disorders: An Overview from the Glia Perspective. Front Integr Neurosci. 9:65

45. World Health Organization (2004): Neuroscience of psychoactive substance use and dependence. WHO Library Cataloguing-in-Publication Data. ISBN 9241562358.

46. Xu Z, Chang LW, Slikker W, Ali SF, Rountree RL, Scallet AC. 2000. A Dose-Response Study of Ibogaine-Induced Neuropathology in the Rat Cerebellum. Toxicol Sci 57:95-101 